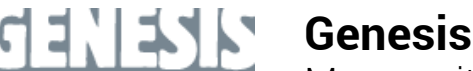

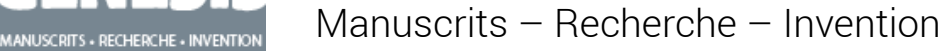

$51 \mid 2020$

Intertextualité - Exogenèse

\section{La Représentation du Discours Autre : principes pour une description, par Jacqueline Authier-Revuz}

Claire Doquet

\section{OpenEdition}

1 Journals

Édition électronique

URL : https://journals.openedition.org/genesis/5568

DOI : 10.4000/genesis.5568

ISSN : 2268-1590

Éditeur :

Presses universitaires de Paris Sorbonne (PUPS), Société internationale de génétique artistique littéraire et scientifique (SIGALES)

Édition imprimée

Date de publication : 15 décembre 2020

ISBN : 979-10-231-0704-3

ISSN : $1167-5101$

\section{Référence électronique}

Claire Doquet, "La Représentation du Discours Autre : principes pour une description, par Jacqueline Authier-Revuz ", Genesis [En ligne], 51 | 2020, mis en ligne le 10 janvier 2021, consulté le 07 février 2022. URL : http://journals.openedition.org/genesis/5568; DOI : https://doi.org/10.4000/genesis. 5568 
nécessaire : il fallait adhérer tacitement à un Credo dont un des articles était que le jeune pianiste, protégé par Mme Verdurin cette année-là et dont elle disait : "Ça ne devrait pas être permis de savoir jouer Wagner comme ça!", "enfonçait" à la fois Planté et Rubinstein et que le docteur Cottard avait plus de diagnostic que Potain.» Voici l'incipit d'Un amour de Swann. Si c'est bien Proust qui tient la plume, qui entend-on dans ces quelques lignes? Le narrateur, bien sûr. Madame Verdurin, avec son langage où se donnent à voir sa vulgarité et sa bêtise. Mais aussi un collectif, qui se désigne - ou que d'autres désignent - comme un «petit noyau», un «petit groupe», un «petit clan », et qui s'accorde à considérer que son jeune pianiste «enfonce»- le mot lui-même compte - les musiciens les plus prestigieux. Dans cet incipit, un narrateur évoque des lieux et des personnages, avec ses propres mots mais surtout avec d'autres mots, venus d'ailleurs et rendus visibles par un système de représentation spécifique. C'est ce système, linguistique, que met au jour l'ouvrage présenté ici.

Linguiste, professeure émérite à la Sorbonne Nouvelle, Jacqueline AuthierRevuz inscrit ses travaux dans la linguistique de l'énonciation. On lui doit, entre autres, le concept de «Représentation du Discours Autre» (RDA), qui fait aujourd'hui l'objet d'un ouvrage magistral où l'autrice montre en plus de 700 pages qu'il est inscrit dans la langue, au sens saussurien du terme, et régi par un système de distinctivité spécifique qui assure son opérationnalité aux plans subjectif et énonciatif. L'étude exhaustive des formes de RDA, appréhendées du triple point de vue syntaxique, sémantique et énonciatif, rend visibles des strates de l'énonciation que la RDA découpe. En appui sur la notion d'hétérogénéité, Authier-Revuz parvient à faire émerger sa «fonction configurative» : configuration du discours, mais aussi, fondamentalement, configuration du sujet. Décrite comme le «nouage de la réflexivité et de l'altérité» (p. XVIII), la RDA est présentée en cinq développements articulés : les trois premiers s'attachent à la caractériser comme une activité métalangagière qui articule le dire représenté et le dire représentant à l'aide de trois opérations; le quatrième expose les cinq «modes» de RDA proposés par la langue; le cinquième est une réflexion sur la configuration subjective que permet la RDA à travers sa fonction de marquage de l'intériorité/extériorité et son «travail des bords ».

Il serait illusoire de vouloir présenter exhaustivement un ouvrage aussi ample, d'une densité et d'une précision rares, et dont la portée heuristique, saillante à la lecture, mettra sans doute plusieurs années, voire décennies, à se déployer dans l'ensemble des sphères concernées par le domaine. Je résumerai rapidement - et par conséquent partiellement - quelques-uns de ses traits les plus vifs pour me concentrer ensuite sur certaines propositions, à mes yeux essentielles, en particulier dans le domaine de l'approche linguistique de l'écriture et des études génétiques.

\section{Du Discours Rapporté à la}

Représentation du Discours Autre : déplacements conceptuels et élargissement du champ de l'étude

En renonçant à l'appellation traditionnelle de Discours Rapporté au profit de la Représentation du Discours Autre, Jacqueline Authier-Revuz déplace la problématique d'un discours que l'on rapporte - et dont elle précisait dès $1992^{1}$ que ce n'est jamais un discours seul mais son énonciation qui est rapportée - en celle d'un discours que l'on représente en tant qu'autre. Ce saut notionnel a des conséquences importantes sur la délimitation du champ comme sur les modalités de sa description.

Le Discours Autre dont il est question dans la RDA n'est pas réductible au Discours d'Autrui. Dans bien des exemples, souvent littéraires, analysés dans l'ouvrage, le locuteur du discours en train de se faire

(*) Université Sorbonne Nouvelle

1. Jacqueline Authier-Revuz, «Repères dans le champ du discours rapporté (suite)», L'Information grammaticale, no 56, p. 10-15. "petit groupe", du "petit clan" des Verdurin, une condition était suffisante mais elle était 
et celui du discours autre représenté sont la même personne, mais dans des conditions et à des époques différentes. Est donc inscrite dans la RDA la représentation de discours de soi plus anciens, ou à venir, en tout cas disjoints de l'ici-maintenant de l'énonciation en cours : si je dis «Tous les jours, je me répétais que j'avais de la chance», c'est de la RDA. En revanche, un énoncé tel que «je te dis qu'il va venir», où l'énoncé métadiscursif (je te dis) et le discours représenté (il va venir) sont référentiellement superposés, relève non de la RDA mais de l'Auto-Représentation du Discours (ARD) (chap. I). L'ARD représente l'énonciation en cours, tandis que la RDA fait advenir dans le dire, «par la représentation qu'elle en donne» (chap. II, p. 38), des événements d'énonciation qui lui sont extérieurs. L'ARD et la RDA ont en commun la réflexivité : une énonciation est représentée en tant que telle; la RDA y ajoute l'altérité : c'est en tant qu'extérieure au dire que cette énonciation est représentée. Derrière la diversité des formes étudiées, elle circonscrit et unifie un type spécifique de pratique discursive qui consiste à inscrire de l'autre dans son discours à différents niveaux.

La Représentation du Discours Autre peut être assurée par les traditionnels Discours Direct, Discours Indirect et Discours Indirect Libre, mais elle ne s'y limite pas. Authier-Revuz y ajoute les modalisations énonciatives, évoquées infra, qui associent le discours autre au propos tenu et en situent la source. Elle inclut dans sa description la catégorisation métalangagière assurée par les désignateurs et introducteurs du discours autre (chap. VI), dont les verbes de parole sont un exemple. L'ensemble des éléments étudiés concourt à l'identification de la RDA comme «pratique métadiscursive spécifique » permettant de «représenter un autre acte d'énonciation", et constituant de la sorte un «métadiscours ordinaire sur l'énonciation» (chap. II). L'autrice met en évidence, entre autres éléments originaux, deux couches dans l'ancrage énonciatif: la couche primaire, celle des déictiques de personne et des temps verbaux ou encore de la modalité énonciative, éléments fondamentaux toujours repérés dans les coordonnées énonciatives du discours qui les porte et en formant «l'assiette énonciative»; la couche secondaire, celle des déictiques spatiotemporels et d'éléments ponctuels de marquage de la subjectivité comme les jurons, labile et susceptible d'un repérage dans les coordonnées énonciatives discours représenté. Dans de multiples exemples, le plus souvent littéraires, elle analyse précisément (chap. IV) ce mécanisme fondamental de la RDA qu'elle utilisera pour en caractériser les modes.

\section{Cinq «solutions» de la langue pour représenter le Discours Autre}

L'étude des moyens linguistiques de la Représentation du Discours Autre permet à l'autrice de dégager les traits discriminants qui aboutissent à la mise en évidence de cinq « solutions » linguistiques, ou «modes de RDA», exclusifs les uns des autres.

Le premier trait distinctif concerne l'articulation des actes d'énonciation du discours en train de se faire (A) et du discours autre représenté (a); on trouve :

1. Des modes à ancrage énonciatif unique : le discours représenté est intégré, énonciativement, dans les coordonnées du discours en train de se faire : le DI et les Modalisations par Discours Autre déjà évoquées.

2. Un mode à deux ancrages énonciatifs hiérarchisés : le DD, qui fait surgir dans le dire une séquence énonciativement hétérogène au dire en cours. Authier-Revuz présente une analyse extrêmement heuristique (chap. IV, p. 123 sq.) de la co-présence des énoncés $E$ (énoncé en cours) et $e$ (énoncé représenté) en expliquant que $e$ est donné comme une «image» d'une chaîne énoncée ailleurs par un locuteur 1, cette «image» étant, dans le cas du DD, énoncée par le locuteur $\mathrm{L}$ du discours représenté.

3. Un mode à ancrage énonciatif partagé : le Discours Indirect Libre, renommé «Bivocal». Au rebours des représentations les plus courantes, pour lesquelles le DIL est envisagé comme forme hybride entre DI et DD ou variante du DI libre de rection, Authier-Revuz le pose comme une forme originale que définit une «bivocalité radicale» (chap. IV, p. 131), manifestée par la co-présence des ancrages énonciatifs du discours en cours et du discours représenté. Plus précisément, le Bivocal-DIL est le seul mode où la couche primaire de l'ancrage énonciatif (cf. supra) peut être fracturée, avec par exemple des pronoms personnels renvoyant au locuteur du discours en cours et une modalité énonciative qui est celle du discours représenté. Cette fracture de la couche primaire, qui casse l'assiette énonciative, explique l'impression d'hybridation produite par le DIL et donnée comme explicative par certains auteurs. L'analyse produite ici formule une position autre, avec des éléments précis et des arguments solides.

Le deuxième trait distingue les modes de RDA selon que le discours autre soit objet ou source du dire. Les traditionnels Discours Direct, Discours Indirect et Discours Indirect Libre servent à la prédication d'un fait de discours autre (exemple : «Marie dit que Lucie viendra»). Un autre moyen de le signaler, la Modalisation, peut porter sur le contenu du Discours Autre (exemple : «D'après Marie, Lucie viendra» où le locuteur, s'énonçant lui-même, mentionne la source de son affirmation) ou sur sa forme (exemple : «Lucie viendra très certainement, pour parler comme Marie», où le locuteur représente l'origine des mots qu'il utilise). C'est à la modalisation portant sur la forme du Discours Autre, ou Modalisation Autonymique, qu'était consacré le premier ouvrage de Jacqueline Authier-Revuz, Ces mots qui ne vont pas de $s o i^{2}$, centré sur les moyens dont dispose la langue pour matérialiser l'hétérogénéité du dire par l'usage des mots comme à la fois désignant un référent et faisant obstacle à cette désignation. Dans l'exemple

2. Jacqueline Authier-Revuz, Ces mots qui ne vont pas de soi: boucles réflexives et noncoüncidences $d u$ dire, 2 vol., Paris, Larousse, 1995 ; 2e édition revue, 1 vol., Limoges, Lambert Lucas, 2012. 
ci-dessus, «Lucie viendra très certainement, pour parler comme Marie», le segment souligné apparaît dans sa matérialité tout en continuant - c'est le propre de ce type d'énoncé - à désigner du hors-langage. Dans le DI, le DD et le Bivocal-DIL, le Discours Autre est l'objet du dire, ce dont on parle (Jean a dit: «c'est nul». / Jean a dit que c'était nul. / Jean s'interrogeait : tout cela était nul); dans les modalisations par discours autre, il est source du dire, ce d'après quoi on parle (D'après Jean, tout ça est nul / Tout ça est «nul», comme dirait Jean).

Le troisième trait distinctif est le caractère autonyme ou non du discours autre représenté. Le chapitre VIII est entièrement consacré à la question de l'autonymie dans le champ de la RDA, distinguant «l'autonymie stricte» du DD de la modalisation autonymique comme «mode dédoublé opacifiant de dire».

Chaque mode de RDA est caractérisé par une formule fondée sur ces trois traits distinctifs : parler $d u$ discours autre ou d'après lui, avec ou sans autonymie, les ancrages énonciatifs des discours en cours et représenté étant unifiés, disjoints ou partagés. Aux formules des cinq modes correspondent des formes linguistiques par lesquelles les modes peuvent se réaliser, l'ensemble caractérisant la RDA comme secteur spécifique de l'activité métalangagière structuré en langue par un système différentiel de signes.

\section{La fonction reconfigurative de la RDA}

Ce troisième aspect de l'ouvrage, auquel est consacrée la cinquième et dernière partie (chap. X à XV), dépasse les investigations linguistiques pour aborder des champs connexes, philosophie et psychanalyse en particulier. Il s'agit de considérer le rôle de la RDA du point de vue de la construction du sujet énonciateur, et d'en caractériser les fonctions. La RDA est envisagée dans son milieu d'exercice, tout à la fois comme rendant visible un ailleurs discursif qu' elle circonscrit et traçant, autour de cet ailleurs, la zone du dedans discursif du sujet, délimitation qui réassure, par contraste, une parole « de soi».

Cette caractérisation différentielle et dialogique de la parole repose sur le versant représenté de l'altérité langagière; celui-ci ne se confond pas avec ce que l'autrice a nommé dès $1982^{3}$ l'hétérogénéité constitutive du dire. Les travaux de Bakhtine, ceux de Pêcheux ont mis en évidence la préséance pour tout dire d'une discursivité qui le nourrit, l'informe, mais aussi le déporte de lui-même; la présence dans toute parole d'un ailleurs discursif tisse le dire d'extériorité. Sur le fond de cette «hétérogénéité constitutive», la RDA apparaît comme un prélèvement opéré métadiscursivement par le sujet parlant dans l'altérité qui traverse ses mots. Ce geste énonciatif de prélèvement dans l'ailleurs constitutif du discours opère un tracé de frontière qui institue différentiellement le complémentaire comme étant de soi. Ici apparaît la fonction positive de configuration du dire par la RDA : le tracé par lequel elle découpe la parole entre ce qui est de l'autre et ce qui n'en est pas soutient la subjectivité du sujet et son sentiment de tenir une parole propre.

La question de la parole propre occupe le chapitre XIII de l'ouvrage. Sur la base de travaux à dominante psychanalytique, l'autrice rappelle la nécessité pour le sujet de se distinguer comme un et de poser entre soi et l'extérieur des limites identifiantes. C'est dans ce cadre qu'apparaît l'importance du sentiment de la parole propre qui structure l'unité du sujet constitué, pour reprendre Benveniste, «dans et par le langage 4 », c'est-à-dire au moyen d'une entité foncièrement étrangère qui, telle une greffe, transforme en sujet humain l'enfant originellement non parlant. Cette appropriation par l'enfant de la langue est fondamentalement contradictoire : elle repose sur la conversion d'une extériorité du langage en parole de soi, sur la nécessité d'énoncer comme de soi des mots qui, tout à la fois venus d'ailleurs et chargés d'une histoire discursive, ne peuvent être à soi. C'est dans cette contradiction que la RDA va, dit l'autrice, «jouer sa partie» (chap. XIV, p. 545) pour permettre au sujet de délimiter sa parole dans la vaste étendue du langage. La RDA est présentée comme "peau du discours », interface entre un intérieur du sujet - qu'il protège - et l'extérieur. AuthierRevuz propose d'aborder les discours par leur RDA, caractérisée comme «mécanisme énonciatif permettant au Discours de se poser comme propre dans l'extériorité en se bordant par une zone de figuration de ses rapports avec d'autres discours qui, comme une peau, le délimite et le protège» (p. 567). $\mathrm{Ce}$ «travail des bords» (chap. XV) est finalement posé comme déterminant des genres de discours, et décrit systématiquement. L'autrice dégage, par exemple : (1) des genres «de RDA», littéraires (la parodie, où le discours fonctionne intégralement en référence à un autre discours) ou non (des discours «tenant lieu» d'un autre discours, tel le procès-verbal qui, une fois signé, vaut pour les propos tenus dans la séance dont il rend compte et constitue une RDA de rang textuel; (2) des genres exempts de RDA, discours de type monologique sans référence aucune à de l'autre (recettes et modes d'emploi, manuels scolaires, articles de loi...); (3) entre les deux, un espace variationnel où il est possible de situer tout discours selon l'étendue de la RDA qu'on y décèle.

L'ouvrage explore d'autres facteurs, qu'il serait trop long d'exposer ici. Je veux simplement saluer la nouveauté du propos et sa fécondité, puisque la RDA, outillée par les moyens finis de son expression mis à disposition par la langue, devient un élément fondamental de différenciation des productions discursives, éclairant leur statut générique mais aussi la caractérisation singulière des écritures.

3. Jacqueline Authier-Revuz, «Hétérogénéité montrée et hétérogénéité constitutive : éléments pour une approche de l'autre dans le discours », DRLAV, no 26, 1982, p. 91-151.

4. «Nous n'atteignons jamais l'homme séparé du langage. [...] C'est dans et par le langage que l'homme se constitue comme sujet» (Émile Benveniste, Problèmes de linguistique générale, Paris, Gallimard, coll. «Bibliothèque des sciences humaines », 1966, p. 259. Cité par Jacqueline Authier-Revuz, p. 503). 
Que peut dire cet ouvrage à la génétique? En quoi le système qu'il met au jour est-il prometteur pour outiller linguistiquement les analyses des manuscrits?

Tout d'abord, et de manière très générale, La Représentation du Discours Autre : principes pour une description précisera à n'en pas douter le cadre théorique du dialogisme exploité, dans ce numéro même, pour décrire la genèse d'un texte comme interaction entre (1) un déjà écrit et (2) la poursuite de son écriture ainsi que sa réécriture, où le scripteur reprend son texte dans une (re)lecture décentrée de l'écriture première et où il le retouche, le prolonge, le corrige... La re-familiarisation nécessaire à la remise au travail quand il s'agit d'écriture est une nouvelle entrée dans l'univers du texte par son auteur qui le (re)lit et doit se le réapproprier avant de poursuivre sa tâche. Une des questions importantes que pose l'ouvrage d'Authier-Revuz à la génétique est de savoir dans quelle mesure et à quelles conditions un scripteur revenant sur son travail a affaire à un discours autre. Le geste de rectification repose sur au moins une des deux dimensions fondamentales de la RDA : la réflexivité. Quid de l'altérité ? Les notions génétiques d'auteur-scripteur et d'auteur-lecteur, et le «troisième rôle» dégagé par Lebrave, celui de la réflexion, peuvent-ils être décrits à travers le prisme de la RDA ? Le paradigme de la représentation du discours autre viendrait alors s'articuler aux concepts énonciatifs déjà utilisés par les généticiens linguistes, par exemple l'ajustement théorisé par Culioli5.

Le déplacement du Discours Rapporté en Représentation du Discours Autre, et le constat selon lequel tous les modes de RDA ont des formes non marquées, outille l'étude de ce qui relève, dans les manuscrits, de l'exogenèse, pour caractériser énonciativement le trajet et la reconfiguration des éléments prélevés ailleurs puis intégrés dans le texte. Chez certains auteurs, par exemple Zola, des écrits intermédiaires balisent le parcours entre les sources externes et la textualisation finale 6 et le marquage métadiscursif, plus ou moins présent, peut être décrit. Chez d'autres, le fil est plus ténu, comme le montre Nathalie Mauriac 7 dans son «enquête génétique» sur un extrait de La Prisonnière où elle met au jour une source journalistique, masquée par le processus d'éclatement et de dissémination qui caractérise l'écriture proustienne mais dont la référence figure dans une note de régie. Elle parle d'allusion pour désigner la présence de ce discours autre dans le texte de Proust, et de fait c'est bien un trajet vers l'allusion - au sens que lui donne Authier-Revuz - qui est effectué, avec la «métabolisation» du discours autre d'abord référencé métadiscursivement avec son titre, puis intégré et transformé au point que le lecteur contemporain ne peut l'identifier.

Il me semble également que les cinq «solutions» offertes par la langue pour représenter le discours autre sont un prisme efficace pour explorer les œuvres, et singulièrement leur écriture : quel mode de RDA domine chez tel auteur, comment évolue la RDA au cours de l'écriture de l'œuvre, quelles substitutions de modes sont-elles repérables? Authier-Revuz mentionne cette piste, qu'ont explorée deux articles récemment parus dans Genesis : dans son étude du discours direct dans les manuscrits de la Comtesse de Ségur, Catherine Boré 8 met au jour un déplacement du DD classique vers le dialogue théâtralisé; Jean Kaempfer et Joël Zufferey ${ }^{9}$ caractérisent la réécriture postéditoriale du roman de Luc Lang Voyage sur la ligne d'horizon par le passage du DD au DDL - article dans lequel les auteurs utilisent d'ailleurs le travail de l'autrice. Ces tentatives s'inscrivent dans ce que l'on pourrait appeler une génétique de la RDA dans l'endogenèse des œuvres étudiées, où se marque la pertinence de la distinction entre RDA et ARD (Auto-Représentation du Discours) : le discours de soi devient autre dès lors que l'auteur reprend un texte sur lequel il a travaillé dans un espace-temps disjoint de celui où il se (re)met à écrire, que l'écart qui sépare l'écriture initiale de sa reprise soit de quelques heures (pour Ségur) ou de plusieurs années (Lang). Les substitutions qui s'effectuent dans la toute proximité de l'énonciation en cours peuvent sans doute se décrire comme des ajustements successifs qui, affectant l'énoncé en train de se constituer, s'apparentent davantage à l'ARD. Cet éclairage énonciatif sur le contexte des substitutions permet de reconsidérer l'articulation entre les variantes de lecture et les variantes d'écriture, catégories héritées de la philologie et qui restent insatisfaisantes dans bien des cas.

La mise au jour de genres de discours globalement métadiscursifs ouvre le champ à des investigations génétiques qui seraient, elles aussi, fondées sur la RDA : peut-on dégager, pour un genre discursif relevant intégralement de la RDA (la parodie, par exemple, ou bien le procès-verbal) des régularités scripturales? Comment cette RDA non marquée se forge-t-elle? Y a-t-il des étapes caractérisées par leur degré de marquage de l'ailleurs discursif qui se trouve représenté?

Enfin, parce qu'il faut conclure, la caractérisation de la RDA comme «travail des bords» permet de se demander, pour toute opération d'écriture, quels bords de l'œuvre on travaille. Lorsque l'auteur rejette un mot (ce que Daniel Ferrer a comparé à la polémique cachée chez Bakhtine ${ }^{10}$ ), il en fait définitivement un discours autre, le plaçant hors de l'œuvre, et du même mouvement délimitant son discours propre. Que produiraient le recensement

5. Antoine Culioli, Pour une linguistique de l'énonciation: opérations et représentation, $\mathrm{t}$. I, Paris, Ophrys, 1990, p. 65 : «à une linguistique de la communication manifeste et réussie, où l'on ne programme que des prédications heureuses», l'auteur préconise de «substituer une linguistique des ajustements énonciatifs».

6. Olivier Lumbroso, Zola autodidacte. Genèse des euvres et apprentissages de l'écrivain en régime naturaliste, Genève, Droz, 2013.

7. Nathalie Mauriac Dyer, «Bidou, Bergotte, la Berma et les Ballets russes », Genesis, no 36 , 2013, p. 51-62, en ligne sur openedition.org.

8. Catherine Boré, «La Comtesse de Ségur ou l'universel du dialogue », Genesis, no 48, 2019 , p. 37-54, en ligne sur openedition.org.

9. Jean Kaempfer et Joël Zufferey, «Luc Lang, récrivain», Genesis, no 50, 2020, p. 133-145, en ligne en août 2021 sur openedition.org.

10. Daniel Ferrer, «Quelques remarques sur le couple énonciation-genèse», Texte, no 27-28, 2000, p. 7-24, en ligne sur item.ens.fr. 
et la mise en lien de tout ce qu'un auteur a supprimé pendant son écriture ? De ce qu'il a ajouté, des remplacements effectués ? C'est à ce type de recensement, aboutissant à un dictionnaire des variantes, que s'est livré Jean-Louis Lebrave dès 1987 dans sa magistrale thèse d'État ${ }^{11}$. Il a analysé la dynamique des variantes en retraçant le parcours scriptural vers ce qui demeurera dans l'œuvre. C'est au trajet inverse que nous convie la perspective différentielle à l'œuvre dans l'ouvrage d'Authier-Revuz. Modifier son écrit, c'est à la fois circonscrire ce qui sera affiché comme de soi et rejeter le reste comme autre. Ce rejet, résultat d'une activité métadiscursive où la réflexivité, quoique non verbalisée, est mise en jeu de manière comparable à ce que révèle la modalisation autonymique, a pour objet un segment de discours qui, examiné pour lui-même, constitue une trace de cette altérité. L'étude de l'ensemble des mots rejetés pourrait ainsi permettre à la fois de retracer le geste de contour par lequel un scripteur délimite son discours, et de décrire l'ailleurs qu'il choisit d'éliminer et sur le fond duquel l'œuvre s'est élaborée.

11. Jean-Louis Lebrave, Le Jeu de l'énonciation en allemand d'après les variantes manuscrites des brouillons d'Henri Heine, thèse de doctorat d'État, 1987, Université de Paris-Sorbonne.

* Université de Lorraine. 